Voix et Images

voixetimages

\title{
Margaret Atwood et Nicole Brossard : la question de la représentation
}

\section{Louise Milot}

Volume 11, numéro 1, automne 1985

Naïm Kattan

URI : https://id.erudit.org/iderudit/200537ar

DOI : https://doi.org/10.7202/200537ar

Aller au sommaire du numéro

Éditeur(s)

Université du Québec à Montréal

ISSN

0318-9201 (imprimé)

1705-933X (numérique)

Découvrir la revue

Citer cet article

Milot, L. (1985). Margaret Atwood et Nicole Brossard : la question de la représentation. Voix et Images, 11(1), 56-62. https://doi.org/10.7202/200537ar d'utilisation que vous pouvez consulter en ligne.

https://apropos.erudit.org/fr/usagers/politique-dutilisation/ 


\title{
Margaret Atwood et Nicole Brossard: la question de la représentation*
}

\author{
par Louise Milot, Université Laval
}

Dans une scène de Marquée au corps, de Margaret Atwood, l'héroïne, Rennie Wilson, déboutonne son corsage, sort son bras de la manche gauche et fait glisser la bretelle de sa combinaison:

- Diable, que faites-vous là? lui dit le gros policier qui

l'interroge,

- Je veux que vous me croyiez, répond-elle ${ }^{1}$.

La mise en discours de cet épisode semble vouloir représenter le malentendu profond qui existe entre Rennie et le policier. Elle, jeune femme vivant seule, obligée de se justifier parce qu'un rôdeur a pénétré chez elle en son absence; lui, imbu de préjugés et responsable de l'enquête. Malentendu d'autant plus lourd à dissiper que le policier prétend, d'un air suffisant, qu'il connaît bien les femmes seules, leurs attitudes provocatrices, leur habitude de ramener des hommes chez elles, et quoi encore ${ }^{2}$. Que Rennie soit placée dans l'obligation d'exhiber la cicatrice d'une toute récente opération à un sein, pour qu'on ne croie pas qu'elle ramène des hommes chez elle, voilà une mise en scène dont l'effet persuasif est aussi puissant sur la lectrice (et le lecteur!) que sur le policier qu'il s'agissait, dans la fiction, de convaincre.

Du point de vue de la représentation de la femme, le seul que nous retenions ici, le roman de Margaret Atwood parvient certes, dans des raccourcis dont l'épisode ci-dessus n'est qu'un exemple, à exprimer la chose suivante: au-delà de sa carrière et de son apparente libération, la journaliste "dans le vent" qu'est Rennie Wilson vit une situation personnelle de total désarroi. Des trois relations qu'elle établit avec des partenaires masculins son amant du début, le médecin qui l'opère, le militant de l'île SaintAntoine - il faut comprendre que chacune, en son temps, est vitale autant qu'inadéquate, et exige de Rennie qu'elle accepte le compromis, l'hiatus, le fait d'être toujours l'accusée:

- Tout le monde rêve, dit-elle un jour à un de ses partenaires,

Pourquoi les hommes ne veulent-ils jamais en parler?

Et la réponse de celui-ci:

C'est pour cela que je ne pouvais pas supporter les États-Unis.

Quand j'y suis retourné, les femmes parlaient comme ça.

Elles commençaient leurs phrases par: Pourquoi

les hommes ne...? ${ }^{3}$.

Mon propos n'est pas d'insister sur les divers effets de sens, ou sur l'effet de sens général du roman de Margaret Atwood. D’ailleurs, le choix de cette auteure, de ce roman, comporte une bonne part d'arbitraire. Il s'agit en fait

\footnotetext{
* Ce texte est la version écrite d'une communication faite à l'APFUCC, Congrès des
} Sociétés savantes, Guelph, juin 1984. 
de mettre l'accent sur un type de texte de facture plutôt conventionnelle, dans lequel est directement perceptible une certaine représentation du monde (liée à un type précis de femme canadienne). Mais Margaret Atwood a produit un texte de fiction dont la facture, au-delà de l'émotion suscitée par le contenu, n'étonne ni ne heurte.

Une telle manière de classer est évidemment discutable; il faut la définir, en tout cas, par rapport à la notion même de texte, plus précisément à la notion de récit de fiction, en regard d'un développement soi-disant moderne de l'écriture. Dans le cadre du parti-pris théorique que nous proposons ici, le discours de la prose fictionnelle, pas plus que le discours pictural, musical ou poétique, n'auraient pour intentionnalité ${ }^{4}$ ce qu'il est convenu d'appeler la représentation du monde: sa seule trajectoire serait son propre développement discursif.

De ce point de vue, l'écriture de Margaret Atwood ne se donne pas pour autre chose que ce qu'elle est: un texte qui porte l'hypothèque du privilège accordé au souci de représenter. Même s'il est sûr que Margaret Atwood fait autre chose que simplement reproduire des situations antérieurement connues ou vécues, même si toute production textuelle comporte inévitablement un travail de construction, il faudrait voir Marquée au corps - sans que cela ne soit péjoratif - comme faisant partie de ces romans qui n'hésitent pas à utiliser et à exploiter ce qui, pour un certain public lecteur, est un attrait aussi indispensable qu'infaillible: les pouvoirs de l'illusion référentielle. En mettant en discours des situations qui semblent avoir été vécues, ou tout au moins qui auraient pu l'être, en mettaint en scène des personnages qui vivent ce que vivent, au même moment, beaucoup de lectrices et de lecteurs, un roman comme Marquée au corps évoque, notamment au sujet des femmes, quelque chose de partiellement connu, mais dont on peut croire qu'il n'est pas inutile de le redire. Il reste qu'il ne viendrait à l'idée de personne de voir dans cette écriture le lieu d'une avant-garde.

Pour cela, on a plutôt l'habitude de se tourner, au Québec tout au moins, vers une auteure comme Nicole Brossard. En effet, du point de vue de l'audace, de l'envergure et de la spécificité même du projet d'écriture, on peut croire que les positions de Nicole Brossard sont ambitieuses, puisqu'il s'est agi pour elle, dès le début -- et ici n'est prise en compte que sa prose - d'une transformation $d u$ langage et, si on croit au pouvoir révolutionnaire des mots, d'une transformation par le langage.

À ce niveau, le travail de Nicole Brossard a été lu par la critique (je pense en particulier à des analyses de Sold Out ${ }^{5}$ ) comme recoupant tout à fait le travail sur le texte préconisé dans les années soixante par Jean Ricardou et ses émules. Cela, indépendamment du fait qu'une telle intertextualité théorique ait été souhaitée, ou pas, chez Brossard.

Pour le groupe des premiers nouveaux romanciers français, la thématique et, d'une certaine façon, le contenu d'un texte sont absolument seconds; s'ils peuvent être débusqués au terme d'une patiente analyse, ils restent d'abord et avant tout la résultante du travail de l'écriture, et ne sont jamais sa raison d'être. Le point de départ d'un roman, dans cette optique, peut être 
purement prétexte: prétexte significatif et signifiant, sans doute, mais prétexte quand même. Le cas-limite serait de partir de rien, entendez du mot rien, mot de quatre lettres dont la démonstration a été faite, à l'époque, qu'il pouvait constituer le premier générateur et ainsi l'explication ultime d'un roman ${ }^{6}$.

Le problème posé, on le voit, est celui du rapport au référent. Préexiste-til au texte et celui-ci tend-il à le traduire, comme il semble que ce soit le cas dans la prose de Margaret Atwood? Est-il au contraire produit et généré par le travail de l'écriture, comme semblent l'attester des romans comme Un livre, Sold Out, French Kiss ${ }^{7}$ ?

C'est à dessein que sont cités des romans relativement anciens de Nicole Brossard. Car mon hypothèse de travail concerne justement ce qui serait une déviance progressive de la prose de Nicole Brossard, provenant de l'importance grandissante accordée, d'un roman à l'autre, à ce que j'appellerai "l'engagement féministe». Indirectement, «la nouvelle écriture» québécoise, vu le rôle central qu'y a joué Nicole Brossard, aurait été aussi marquée par cette déviance.

Cette manière de voir semble illustrée avec assez de clarté par Picture Theory, paru en $1982^{\circ}$, particulièrement par la finale en apothéose, par le commentaire de Nicole Brossard elle-même sur le sens de cette finale et, de façon générale, sur le sens du roman pris globalement.

Dans une entrevue accordée à la Nouvelle Barre du jour ${ }^{9}$, Nicole Brossard n'a laissé aucun doute sur sa certitude d'être arrivée, dans ce dernier roman, à une synthèse exceptionnelle (absolument inouie, déclare-t-elle), qui n'ouvrira pas sur une autre fiction, mais plutôt sur un essai:

Je pense à un essai sur la modernité, sur l'écriture, sur le

féminisme et le lesbianisme, sur l'imaginaire, la vision aérienne,

l'hologramme, la lumière, etc. (NBJ, p. 201).

Lue par Nicole Brossard elle-même et par la critique comme un lieu d'aboutissement, une réussite de toute l'œuvre en prose depuis 1970, et en même temps comme le point d'ancrage d'essais à venir, la finale de Picture Theory est une charnière qu'il vaut d'examiner. Rappelons que la performance consiste, si on la ramène à l'essentiel, à faire surgir, par le biais de l'hologramme, une femme fictive devenue réelle ${ }^{10}$.

À première vue, nous avons là utilisation d'un procédé résolument "moderne", au sens où nous avons défini ci-dessus la modernité de l'écriture, c'est-à-dire comme une production, par le travail des mots, d'un nouveau sens, la seule réalité à laquelle puisse de toute façon prétendre l'écriture. Ici, le sens correspondrait à cette femme nouvelle. Comme on peut le lire dans Picture Theory, non pas la femme née d'une première femme ou éventuellement de l'image traditionnelle de la mère, mais une femme véritablement autre: celle par qui tout peut arriver"l.

Le procédé peut toutefois être lu autrement. En effet, en dépit d'une structure où il semble que le travail des mots ait fini par engendrer la réalité femme nouvelle, une ambiguïté se dessine, car cet effet de sens n'est pas un produit inattendu du travail de l'écriture, ni même son effet second mais, 
bien au contraire, un effet de sens tout à fait escompté puisque c'est lui, cet effet de sens ultime à obtenir, à atteindre, qui a contraint, en l'occurrence, l'œuvre entière:

Chacune des phrases construit le projet et rend plausible le dernier chapitre qui est l'hologramme. Tout le livre est structuré pour arriver à écrire cet Hologramme (Nicole Brossard à la NBJ, p. 180).

Ainsi, en ce qui concerne le rapport au référent, et même sans tenir compte de formes d'écriture carrément différentes, peut-on ne pas s'inquiéter de parvenir à rapprocher, au niveau de leurs présupposés, des romans comme ceux de Margaret Atwood et de Nicole Brossard?

Il faut bien voir en effet que dans un cas comme dans l'autre, la domination du référentiel et la contrainte que cela implique fonctionne à plein. Que nous ayons affaire à une image de femme qui existe à peu près telle quelle dans la réalité (discours "réaliste» de Margaret Atwood) ou à une image de femme pour le moment impossible (discours de l'«utopie" de Nicole Brossard), le degré et la conception de l'engagement féministe changent mais, d'un point de vue textuel, ne nous ramène-t-on pas à une confortable tradition? Ầ cela même que le discours moderne a tenté d'évacuer: de la poésie, depuis la fin du XIX ${ }^{\mathrm{c}}$ siècle, de la peinture, depuis le début du XX', de la prose, en France, depuis les années 50 ou au Québec depuis les années 60, justement depuis Nicole Brossard.

Tel est le paradoxe. L'auteure québécoise la plus couramment associée aux postulats de la nouvelle écriture, et d'abord accaparée, pouvait-on croire, par les seules aventures de cette écriture, a fini par faire la place la plus grande à la démonstration d'une thématique féministe. La prétention à l'antériorité ou à la primauté de l'écriture, chez Nicole Brossard, s'en trouve affaiblie d'autant. Et on peut se demander si le discours fictif lui-même ne se dirige pas vers une impasse.

Invitée à l'université Laval, en mai 1984, dans le cadre du Congrès de l'ACFAS*, Nicole Brossard y donnait comme justification d'une certaine opacité de sa prose fictionnelle et de son travail (inédit) sur le langage, non pas des motifs théoriques reliés à une conception de l'écriture, mais la nécessité d'une nouvelle langue, d'une langue particulière à la femme et lui appartenant. Une langue nouvelle pour une femme nouvelle, qui affranchirait celle-ci de la domination - même linguistique - des hommes. S'il faut en croire Nicole Brossard, tout son travail de virtuosité linguistique serait à lire en étroite relation avec une telle thématique. La cause est bonne et le projet se défend: mais n'y a-t-il pas risque de confusion? La cause des femmes, c'est une chose; les opérations de décapage nécessaires face à des conceptions vieillies du texte, on peut penser que c'en est une autre. Et ce qui vaut pour la première n'est pas nécessairement un pas en avant pour la seconde.

- ACFAS: Association Canadienne Française pour l'Avancement des Sciences. 
Pourtant, Nicole Brossard unit de nouveau les deux causes, quand elle explique, à la même séance de l'ACFAS, qu'un texte doit procurer à ses lecteurs deux sortes de satisfaction:

a) une satisfaction d'ordre logique, le texte offrant une résistance, présentant une énigme à résoudre: voilà qui répond bien au type de questionnement que pose, en principe, le texte moderne;

b) une satisfaction de l'ordre de l'identification: sur ce point, de plus en plus de "nouvelles lectrices" - Nicole Brossard reconnaît que les lecteurs seront plus longs à y venir - devraient pouvoir se retrouver, idéalement, dans le type de prose qu'elle pratique.

La confusion entre ce qui est pertinent pour la lutte féministe et ce qui l'est pour la lutte textuelle est clairement perceptible ici. Un roman comme Marquée au corps, qui recherche visiblement l'image conforme, suscite à la lecture un processus d'identification. Les analystes de "nouvelle écriture» avaient cru comprendre, cependant, que tout le travail de sape de ces mouvements (face à nos "anciennes" perceptions du rôle de la littérature) tendait, justement, à établir que l'écriture de la fiction avait le pas sur le contenu de celle-ci, que la thématique féministe, par exemple, ne pouvait en aucun cas être reçue comme centrale ou comme responsable de l'écriture.

Depuis les années 1970, beaucoup d'efforts ont été demandés au public lecteur, par Nicole Brossard et par d'autres, au nom de ce que ce public, qui s'est prêté à l'opération de plus ou moins bonne grâce, avait compris être un renouvellement, voire un progrès formel, au niveau du travail du langage. Il est un peu frustrant de constater ou de se faire dire, dix ans plus tard, que tout cet investissement a atteint son but au moment du surgissement, à la fin de Picture Theory, d'une femme fictive, la plus réelle possible. Si on ne peut nier que, de ce fait, la cause féministe se trouve en progression, on est par contre beaucoup moins optimiste quant à ce qui en est de la cause de l'écriture.

Cela suggère au moins deux remarques; la première est assez générale, l'autre nous ramènera à Nicole Brossard.

1. Il y a eu à peu près coïncidence dans le temps, au Québec, entre les revendications des féministes et un certain éclatement formel de l'écriture tendant à imposer, en lieu et place d'une écriture-reflet, disons une écritureproduction. Cette coïncidence a sans doute teinté dans un sens particulier la nouvelle écriture québécoise à laquelle se sont trouvé associées, à tort ou à raison, un fort contingent de femmes et, encore maintenant, de féministes militantes. De la sorte s'est construite comme une équation qu'il serait, je crois, pertinent de désamorcer, entre une forme nouvelle ou déviante d'écriture et un contenu à portée féministe, radical ou pas.

C'est ce que nous proposons de faire, en revendiquant la possibilité de lire la prose de Nicole Brossard comme risquant de nous ramener au piège de la représentation, quel que soit le côté "moderne" de son contenu. C'est dire qu'une littérature engagée, si noble et pertinente que soit sa cause, peut nous reporter bien des années en arrière, du point de vue théorique de la définition 
du texte. Peu importe son habillage formel, nous posons qu'une telle fiction, dont il ne s'agit pas de contester par ailleurs la portée sociale, ne peut faire avancer la cause de la spécificité du textuel que de façon ambiguẻ.

2. Picture Theory - à traduire, selon les indications de l'auteure, par tableau de réalité ou peinture de réalité (NBJ, p. 178) - privilégie en situation finale le procédé mi-réel, mi-fictif de l'holographie. En même temps, ce roman semble marquer une ouverture vers une œuvre clairement militante, une cuvre d'essayiste. Nicole Brossard ne montre-t-elle pas le "danger» que fait surgir pour la fiction, à plus ou moins long terme, ce choix de laisser quelque chose comme le féminisme envahir le terrain du textuel fictif?

Ce choix est évidemment celui de Nicole Brossard. Il n'y a pas lieu de le discuter. Toutefois, pour nous, et c'est en ce sens seulement que nous avons pu parler de déviance, le choix de Nicole Brossard impliquera, au moment de faire le bilan des acquis des quinze dernières années, la nécessité de déterminer quel prix la petite histoire de la nouvelle écriture québécoise aura payé son allégeance à Nicole Brossard.

\section{Pour conclure:}

Si on essaie, au terme de ce parcours, de remettre en perspective les textes de Margaret Atwood et ceux de Nicole Brossard, on peut se retrouver devant des résultats assez surprenants, pour ne pas dire paradoxaux.

Au point de départ, il semblait bien que, des deux textes en présence, celui de Margaret Atwood s'appliquait à dire quelque chose au moyen d'une fiction, celui de Nicole Brossard s'appliquant à faire cette fiction. Une telle façon de voir reste peut-être toujours pertinente pour le roman de Margaret Atwood. Encore que, lorsque Rennie Wilson, forcée d'agir pour faire croire, traduit cet agir en langage - Je veux que vous me croyiez, lui faisait dire le texte - il y a là véritable constitution d'un discours fictionnel par le langage, quelle qu'en soit la part respective de tradition et de modernité.

Quant à Nicole Brossard, qui de plus en plus aurait mis son dire au service d'un faire - son action militante - elle semble en être venue à tout simplement sortir de l'espace strict de la fiction. Et puisque c'est de faire dont l'exigence se faisait surtout sentir, elle s'est vue contrainte, selon ses dires, d'essayer l'essai, en "Claire Dérive" - pour emprunter le nom du personnage de Picture Theory - par rapport au texte fictionnel. 
1. Margaret Atwood, Marquée au corps, traduit de l’anglais par Hélène Filion, Quinze, 1983 , p. 15.

2. Ibid., p. 14

3. Ibid., p. 222.

4. Intentionnalité au sens greimassien, à ne pas confondre avec intention; voir A.J. Greimas et J. Courtés, Sémiotique, Dictionnaire raisonné de la théorie du langage, Hachette, 1979, p. 190.

5. Voir les articles d'Irène Duranleau et de Jean-Marcel Léard, dans Études littéraires, vol. 14 , no. I, avril 1981 .

6. Jean Ricardou, “La fiction à mesure», dans Nouveaux problèmes du roman, Seuil, "Poétique», 1978, pp. 244-351.

7. Nicole Brossard, Un livre, Quinze/présence, 1980;

Sold Out, étreinte/illustration, Quinze/présence, 1980;

French Kiss, Éd. du Jour, 1974.

8. Nicole Brossard, Picture Theory, Nouvelle Optique, 1982.

9. «Entretien avec Nicole Brossard sur Picture Theory réalisé à Montréal le 13 juin 1982", NBJ, 118-119, pp. 177-202.

10. Picture Theory, pp. 173ss.

11. Ibid., p. 165. 